\title{
Response to the Letter on "The Role of Combined SGLT1/SGLT2 Inhibition in Reducing the Incidence of Stroke and Myocardial Infarction in Patients with Type 2 Diabetes Mellitus"
}

\author{
Bertram Pitt ${ }^{1} \cdot$ Gabriel Steg $^{2}$ - Lawrence A. Leiter ${ }^{3} \cdot$ Deepak L. Bhatt $^{4}$ \\ Accepted: 15 December 2021 / Published online: 10 January 2022 \\ (c) The Author(s), under exclusive licence to Springer Science+Business Media, LLC, part of Springer Nature 2022
}

Keywords Type 2 diabetes $\cdot$ Cardiovascular risk $\cdot$ Sodium-glucose cotransporter 1/2 inhibitors

We thank the authors of the letter (DTY-D-21-00785R1) for their interest in our work [1]. As pointed out, the correct reference for the finding of a significant reduction in the incidence of stroke and myocardial infarction (MI) associated with the use of the SGLT1/2 inhibitor sotagliflozin is from the SCORED trial [2] rather than from the SOLOIST trial [3]. We agree that the significant reductions in MI and in stroke (as well as in heart failure) with sotagliflozin were proven in patients with type 2 diabetes mellitus and chronic kidney disease in SCORED and that the significant reduction in heart failure with sotagliflozin in SOLOIST was proven in patients with acute worsening heart failure, but we believe that these benefits may extend to other patients beyond those specifically studied in the trials. However, future trials will be necessary to evaluate sotagliflozin's effect on cardiovascular outcomes in patients without diabetes-in those with or without chronic kidney disease and with or without heart failure.

We disagree with the authors' suggestion that the addition of SGLT1 to SGLT2 inhibition in patients with worsening heart failure could be deleterious, since there were highly significant reductions in the primary endpoint of cardiovascular death, hospitalizations, and urgent visits for heart failure in patients randomized to sotagliflozin in both SCORED and SOLOIST, including in the patients who had evidence

\footnotetext{
Deepak L. Bhatt

DLBhattMD@post.Harvard.edu

University of Michigan, Ann Arbor, MI, USA

2 Université de Paris, Hopital Bichat, Paris, France

3 Li Ka Shing Knowledge Institute, St. Michael's Hospital, University of Toronto, Toronto, ON, Canada

4 Brigham and Women's Hospital Heart \& Vascular Center and Harvard Medical School, 75 Francis Street, Boston, MA 02115, USA
}

of heart failure-either with reduced or preserved ejection fraction. In fact, SCORED and SOLOIST were the first trials in any drug class to demonstrate significant benefit in patients with heart failure with preserved ejection fraction, with benefits across the full range of ejection fraction.

\section{Declarations}

Disclosures Disclosures - as listed in the original article.

\section{References}

1. Pitt B, Steg G, Leiter LA, Bhatt DL. The role of combined SGLT1/2 inhibition in reducing the incidence of stroke and myocardial infarction in patients with type 2 diabetes mellitus. Cardiovasc Drugs Therapy. 2021. https://doi.org/10.1007/ s10557-021-07291-y.

2. Bhatt DL, Szarek M, Pitt B, et al. Sotagliflozin in patients with diabetes and chronic kidney disease. N Engl J Med. 2021;384:129-39.

3. Bhatt DL, Szarek M, Steg PG, et al. Sotagliflozin in patients with diabetes and recent worsening heart failure. $\mathrm{N}$ Engl J Med. 2021;384:117-28.

Publisher's Note Springer Nature remains neutral with regard to jurisdictional claims in published maps and institutional affiliations. 